\title{
8 Die Krim zwischen Ostrom, Krim-Gotthia und dem Chasarenreich
}

\author{
Als der Fürst Svjatosláv herangewachsen und zum \\ Manne gereift war, begann er, Krieger zu sammeln, viele und tapfere, \\ denn er war auch selbst tapfer; und indem er leicht dahinzog, wie ein Panther, führte er viele \\ Kriege. Denn wenn er dahinzog, ließ er keine Wagen hinter sich her fahren noch einen \\ Kessel, noch ließ er Fleisch kochen, sondern sie schnitten ein dünnes Stück Fleisch vom \\ Pferd oder vom Wild oder vom Rind heraus, brieten es auf Kohlen und aßen es. \\ Auch hatte er kein Zelt, sondern breitete die Satteldecke aus und den Sattel zu Häupten, \\ und so waren auch alle seine übrigen Krieger. \\ Und er sandte hin zu den [anderen] Ländern und ließ sagen: Ich werde gegen euch ziehen! \\ Und er zog zum Fluß Oka, und zur Vólga und fand die Vjatičen. \\ Und er sagte zu den Vjatičen: Wem zahlt ihr Tribut? \\ Die aber sagten: Den Chasaren zahlen wir je einen Schilling vom Pflug. \\ Svjatosláv zog gegen die Chasaren. \\ Als aber die Chasaren das hörten, zogen sie aus, ihm entgegen, mit ihrem Fürsten, dem \\ Kagán. \\ Und sie trafen zusammen, [gegeneinander] zu kämpfen. \\ Und als es zur Schlacht kam, gewann Svjatosláv die Oberhand über die Chasaren, \\ und er nahm ihre Stadt Belaja Veza. ${ }^{1}$
}

Der Sieg Svjatoslavs I. (um 942-972), Großfürst des ersten altostslavischen Großreiches der Kiewer Rus', und seiner Krieger über die Chasaren im Jahr 965 wurde in der „Nestorchronik“ mit den obigen Worten beschrieben. Der Fürst wird als genügsam geschildert, er führt das ,soldatische‘, entbehrungsreiche Leben eines klassischen Kämpfers. Es nimmt somit eigentlich kein Wunder, dass er den Chasaren eine Niederlage beibrachte, welche das Ende ihrer seit dem Ende des 7. Jahrhunderts dauernden Herrschaft über weite Teile des nördlichen und östlichen Ufers des Schwarzen Meeres einleitete. Die turkstämmigen Chasaren waren somit für knapp drei Jahrhunderte - neben dem die südliche Krim einschließlich Chersones beherrschenden Ostrom - eine entscheidende regionale Ordnungsmacht gewesen. ${ }^{2}$ Kleinere territoriale Herrschaften wie Krim-Gotthia entrichteten ihnen Tribut, und eine weitere bedeutende Einnahmequelle erwuchs ihnen durch die weitgehende Kontrolle des Fernhandels - einschließlich des Verkaufs von SklavInnen - zwischen dem Schwarzen Meer und Asien. ${ }^{3}$

1 Müller L. (2001), $78 \mathrm{f}$.

2 Vgl. u.a. Pletnjowa (1978); Zhivkov (2015).Vgl. auch die Analyse der Beziehungen zwischen den Chasaren und der Kiewer Rus': Petrukhin (2007).

3 Zur Wirtschaft vgl. Noonan (2007), zur Krim insbesondere 219-228.

๑ OpenAccess. (๑) 2020 Kerstin S. Jobst, publiziert von De Gruyter. [(c))BY Dieses Werk ist lizenziert unter der Creative Commons Attribution 4.0 International. https://doi.org/10.1515/9783110520620-010 
Die Beziehungen zwischen den Chasaren und Konstantinopel - einem weiteren wichtigen Machtzentrum in der Region - waren wechselhaft: Bündnisse gegen eindringende nomadisierende Gruppen wie die Petschenegen oder auch gegen das persische Sassanidenreich wurden fallweise geschlossen und wieder gelöst. ${ }^{4}$ Wie das Kräfteverhältnis zwischen diesen beiden Akteuren gerade in ihren Interaktionen auf der Halbinsel gewesen ist, wird von der Forschung unterschiedlich beurteilt. Gegenwärtig herrscht die Auffassung vor, es habe zumindest phasenweise eine gemeinsame byzantinisch-chasarische Verwaltung über die Krim gegeben. ${ }^{5}$ Das chasarische Großreich ging weit über die eigentliche Halbinsel hinaus und erstreckte sich über die heute südukrainischen und südrussischen Steppen zwischen Dnepr und Wolga bis in den Kaukasus. Das chasarische Chaganat hat immer wieder das Interesse der Forschung auf sich gezogen, da hier der historisch wohl exzeptionelle Fall vorliegt, dass definitiv die Oberschichten und auch Angehörige anderer sozialer Gruppen mehrheitlich dem Judentum angehört haben, eventuell einem „synkretisch verformten. “6 Ein Grund für die Annahme des jüdischen Glaubens war, so zumindest erklärte es 1962 der sowjetische Historiker Michail I. Artamonov (1898-1972) recht plausibel, eine verbindende religiöse Ideologie zu schaffen, die dieser politischen Entität ein Alleinstellungsmerkmal verlieh - gegenüber dem christlichen Byzanz und den in den Kaukasus vordringenden muslimischen Arabern. ${ }^{7}$ Die eingangs beschriebene (vgl. Kapitel 2) vergebliche Missionsreise der sog. Slavenapostel Konstantin und Methodius zur Bekehrung der chasarischen Eliten erfreute sich insbesondere der Aufmerksamkeit der russischen (und sowjetischen) Forschung, da damit eine weitere Möglichkeit gesehen wurde, eine lineare Geschichtsinterpretation über die Verbindung zwischen Schwarzmeerraum und Orthodoxie zu schaffen. ${ }^{8}$ Über die innere Verfasstheit des Chasarenreichs ist an dieser Stelle nur so viel zu sagen, als dass von einem „sakralen Königreich“ zu sprechen ist: Der Chagan (in etwa: Großchan), so hat es zumindest P. B. Golden ausgeführt, herrschte über das Chasarenreich, regierte es aber nicht. Für die allfälligen Regierungsgeschäfte war nämlich ein in den Quellen als Beg, Yilig oder Šad bezeichneter und mit „König“ zu übersetzender Angehöriger eines bestimmten Clans zuständig. In der Hierarchie stand dieser König unter dem Chagan, dem vor allen Dingen eine spirituelle Funktion zukam: „The Qağan is a heavenly mandate intermediary between the

\footnotetext{
4 Noonan (1992).

5 Vgl. dazu Albrecht u. Herdick (2013), 31.

6 Altschüler (2006), 190.

7 Artamonov (1962), $266 \mathrm{f}$.

8 Vgl. einen aktuellen Forschungsüberblick bei Alikberov u.a. (2010). Vgl. auch Bujnoch (1972), besonders 54-106.
} 
divine and this state. “ Die Herrschaft eines Chagan war auf maximal vierzig Jahre beschränkt, da, so glaubte man, nach diesem Zeitraum seine spirituelle Kraft erschöpft war und er abgelöst werden musste. ${ }^{9}$

Die bereits ausführlich beschriebene Rolle der Krim als kulturelle Kontaktzone (vgl. Kapitel 3) und als Exilort an der Peripherie von Imperien oder Großreichen wird am Beispiel des byzantinischen Kaisers Justinian II. (668/669-711) ein weiteres Mal augenfällig. Einer breiteren Öffentlichkeit ist er bis heute vor allen Dingen wegen seiner (freilich nicht von ihm selbst eingeführten) Praxis bekannt, seinen Gegnern die Nase abschneiden zu lassen; ein Schicksal, welches ihm später dann selbst widerfuhr und was ihm den Beinamen „Rhinotmetos“ (etwa: „der mit der abgeschnittenen Nase“) einbrachte. Während er außenpolitisch am Beginn seiner Herrschaft etwa gegenüber den an das Mittelmeer vordringenden Arabern Erfolge verzeichnen konnte, gelang die von ihm gewünschte Wiederannäherung der östlichen und westlichen Kirche nicht. ${ }^{10}$ Innenpolitisch brachte er sowohl die Unterschichten als auch die Eliten gegen sich auf, was 695 zu seinem Sturz, der öffentlichen Abschneidung seiner Nase und - hier kommt die Krim ins Spiel - zu seiner Verbannung in den byzantinischen Außenposten Chersones führte. Sein Bestreben blieb aber die Wiedererlangung des Kaiserthrons, wofür er auch an seinem Verbannungsort agierte. Die „offen hochverräterischen Reden, die er dort führte“11, beunruhigten die Chersoniten dem Anschein nach, die Konstantinopel über Justinians Treiben im Exil informierten. Aus Angst vor einer drohenden Gefangennahme oder Schlimmerem floh der abgesetzte Kaiser 698 oder 704 in die Bergregion der Halbinsel - in die militärisch nur schwer zugängliche Krim-Gotthia (Doros). Nicht restlos geklärt ist, welchen Status Doros zu diesem Zeitpunkt hatte: Stand es bereits unter direkter oder zumindest indirekter Herrschaft der Chasaren, wie es ein Teil der Forschung vermutet, ${ }^{12}$ oder ist Vasil'ev zuzustimmen, der darauf beharrte, dass Doros weder Teil Byzanz' noch des Chaganats war, sondern ein neutrales Gebiet? ${ }^{13}$ Thomas S. Noonan (19382001), der ohne Zweifel einer der profiliertesten Historiker der Geschichte der Chasaren im Schwarzmeerraum war, äußerte sich dahingehend, dass byzantinische Quellen von einer Kontrolle des Chaganats über weite Teile der Krim einschließlich Chersones im 7. Jahrhundert ausgehen, während für das 9. und

9 Golden (2006), 85; etwas abweichend die Interpretation von Altschüler (2006), 237f., der das Chaganat als „Monarchie mit Gewaltenteilung“ bezeichnet, wonach es zwei Chagane gegeben habe, der eine sei für die Legislative, der andere für die Exekutive zuständig gewesen.

10 Zur Biographie Justinians II. vgl. einführend Dieten (1976); Leontsine (2012).

11 Albrecht u. Herdick (2013), 29.

12 Beispielsweise Pletnjowa (1978), 37.

13 Vasiliev (1936), 83. 
10. Jahrhundert keine definitiven Aussagen möglich sind. Einige WissenschaftlerInnen gehen für diesen Zeitraum hingegen von einer abermaligen Dominanz Byzanz' über die Halbinsel aus, ${ }^{14}$ andere halten ein byzantinisch-chasarisches Kondominium über weite Teile der Halbinsel für wahrscheinlich. Für Letzteres spricht, dass diese Praktik durchaus zum byzantinischen Repertoire der Zeit gehörte und beispielsweise zwischen Konstantinopel und den Arabern in Bezug auf Zypern Anwendung fand. ${ }^{15}$

Wie auch immer: Justinian II. hatte sicher nicht ohne Bedacht Doros als Zufluchtsort gewählt, erschien es ihm doch offensichtlich dem Zugriff Byzanz' entzogen. Ihm gelang dort, ein Bündnis mit dem Chagan Busir Glavan (Ende 7./Anfang 8. Jahrhundert; russ./ukr. Ibuzir Gljavan) zu schließen, der ihm zur Bekräftigung dieser Allianz seine Schwester zur Frau gab. Kaiser Tiberios II. (?706, byzantinischer Kaiser 698-705) setzte die Chasaren nun unter Druck, drängte auf Justinians Tötung oder dessen Auslieferung. Daraufhin floh dieser abermals, diesmal nach Phanagoria (auf der Halbinsel Taman), schloss dort einen gegen Konstantinopel gerichteten Pakt mit dem Chan der Protobulgaren, Tervel (700721), und nahm im Jahr 705 Konstantinopel nach mehrtägiger Belagerung ein. Justinian wurde zum zweiten Mal Kaiser. Und er nahm - so will es die Legende Rache an seinen Gegnern, u.a. an Chersones, das sich vorsorglich unter den Schutz von Justinians Schwager Busir Glavan gestellt hatte. Dennoch hatte sich die Lage Justinians nicht wirklich entspannt, denn 711 kam es unter der Ägide eines exilierten Militärs der byzantinischen Armee armenischer Herkunft mit dem Namen Vardanis (als Philippikos Bardanes byzantinischer Kaiser von 711-713) zu einem Aufstand. Mit Hilfe der Chersoniten und der Chasaren ließ Vardanis sich zum Kaiser krönen, segelte nach Konstantinopel - und ließ Justinian II. und dessen noch im Kindesalter befindlichen Sohn töten. ${ }^{16}$ Weder das erste noch das letzte Mal hatte sich das Schicksal von Imperien und ihrer Herrscher also auf der Krim - und damit aus der Perspektive der Metropolen in einer peripheren Weltgegend - entschieden.

Die hervorgehobene Bedeutung der im Südwesten der Krim liegenden Stadt Chersones im Kontext der byzantinischen Reichsstruktur wird am Beispiel der Person Justinians II. und seines Kampfes um den Thron deutlich. Insgesamt ist festzuhalten, dass dieser aus der Perspektive Konstantinopels an der Peripherie gelegene Außenposten des Imperiums von der Antike bis in das 15. Jahrhundert hinein eine große Bedeutung für die Geschichte der Krim und der Schwarz-

14 Noonan, The Economy of the Khazar Khaganate, $219 \mathrm{f}$.

15 Vgl. dazu ausführlich Albrecht u. Herdick (2013).

16 Vgl. dazu u.a. Brook (2006), $136 \mathrm{f}$. 
meerregion insgesamt hatte. ${ }^{17}$ Dies liegt vor allen Dingen an der strategischökonomisch wichtigen Lage der Stadt Chersones und der damit verbundenen Mittlerfunktion zwischen Steppe und Metropole sowie zwischen Sesshaften und nomadischen Kulturen. Ihr kommt zudem nicht nur besondere Relevanz im Kontext der Krim selbst (einschließlich der in Kapitel 2 geschilderten Rolle in einem kulturübergreifenden Mythenbestand) zu, sondern sie zählt auch ohne Zweifel „zu den am besten erforschten byzantinischen Städten“. Chersones ist geradezu „das Beispiel par excellence für eine byzantinische Stadt der ,dunklen Jahrhunderte““, so die Feststellung Wolfram Brandes’, der aber zugleich vor genau diesem Urteil warnt. Er betont vielmehr die Sonderstellung Chersones’ ,am Rande der byzantinischen Kultur und in nächster Nähe zu verschiedenen barbarischen Völkerschaften - meist Reiternomaden“ und den Umstand, dass die archäologische Forschung seit dem ausgehenden 18. Jahrhundert - korrekter wäre das beginnende 19. Jahrhundert ${ }^{18}$ - dort recht energisch vorangetrieben wurde. ${ }^{19}$ Die Kenntnis über die verschiedenen historischen Schichten dieser Stadt ist somit größer als über andere vergleichbare Konglomerate.

Die Bedeutung der Krim insgesamt als Kontaktzone auch zwischen den Religionen zieht sich durch ihre Geschichte und wurde in diesem Buch schon wiederholt thematisiert. Epochenübergreifend versuchten die AkteurInnen grundsätzlich (und schon aus pragmatischen Gründen), die gemeinsame Lebenswelt gewaltarm auszugestalten. Allerdings wurde auch im Fall der Krim der Konsens zwischen den diversen religiösen und konfessionellen Gruppen zuweilen gestört. In diesem Zusammenhang wird in der einschlägigen Literatur immer wieder ein gegen die chasarische Oberhoheit gerichteter Aufstand in den Jahren 786/787 erwähnt, der zugleich Hinweise auf die „Höhen und Tiefen des byzantinisch-chazarischen Verhältnisses in der südwestlichen Krim“ gibt. ${ }^{20}$ Der, wenn man Vasil'ev folgt, erste Bischof von Krim-Gotthia, Johannes $(?-791)^{21}$, soll eine gegen die chasarische Herrschaft gerichtete Revolte angeführt haben. Der auf der Krim geborene Kirchenmann war nach Jahren im Kaukasus wohl um das Jahr 758 auf die Halbinsel zurückgekehrt, was zumeist damit erklärt wird, dass Johannes ein Vertreter der damals dominierenden bilderfeindlichen (= ikonoklastischen) Strömung innerhalb der Kirche gewesen sein soll - und diese Position sollte durch ihn auf der

17 Jobst (2013a).

18 Zur Geschichte und dem Stand der Ausgrabungen: Mack u. Carter (2003). Immer noch wertvoll: Jakobson (1959), für einen Überblick über Grabungen im Zarenreich besonders 5-16.

19 Brandes W. (1988), hier $187 \mathrm{f}$.

20 Albrecht u. Herdick (2013), 31.

21 Vasiliev (1936), 80. 
Krim gestärkt werden. ${ }^{22}$ Dem später heiliggesprochenen Johannes und seinen Anhängern ging es offenbar darum, Doros unter byzantinische Herrschaft zu stellen. ${ }^{23}$ Sie erhielten nicht die Unterstützung Konstantinopels, da der „Kaiser den Status quo auf der Krim offenbar nicht antasten“ wollte. ${ }^{24}$ Dennoch gelang es den Aufständischen kurzzeitig, die chasarische Oberhoheit abzuschütteln und den Tudun (eine Art Statthalter) zu vertreiben. Wenige Monate später kehrte die chasarische Macht jedoch zurück, und die Rädelsführer wurden bestraft bzw. von der Krim entfernt oder konnten fliehen. So heißt es zumindest in der Vita des Johannes ${ }^{25}$, welche zumeist als Grundlage für die Beschreibung dieses Aufstandes genommen wird, wobei aber einige Fragen offen bleiben müssen. ${ }^{26}$

Die chasarische Macht auf der Krim war von Johannes und seinen Mitstreitern herausgefordert worden, ohne dass ihr Einfluss nachhaltig geschwächt worden wäre. Im Verlauf des 10. Jahrhunderts hatte das Chaganat jedoch neuen Eindringlingen aus dem eurasischen Steppenraum (z. B. Alanen und Petschenegen) zunehmend weniger entgegenzusetzen. Aus dem Nordwesten kamen weitere Bedrohungen - nämlich (wie eingangs bereits erwähnt) in Gestalt der Kiewer Rus’, denn der Großfürst „Svjatosláv zog gegen die Chasaren. Als aber die Chasaren das hören, zogen sie aus, mit ihrem Fürsten, dem Kagán. Und sie trafen zusammen, [gegeneinander] zu kämpfen. Und als es zur Schlacht kam, gewann Svjatosláv die Oberhand“, so heißt es in der „Nestorchronik“. ${ }^{27}$ Die zunehmende, auch militärische, Schwäche des Chaganats wurde von Zeitgenossen sehr wohl bemerkt. So wird in der berühmten Lehrschrift „De administrando imperio“, die offenbar in der ersten Hälfte des 10. Jahrhunderts entstanden ist und zumeist dem byzantinischen Kaiser Konstantin VII. (Porphyrogenitus [der Purpurgeborene], 905-959) zugeschrieben wird, der Machtzerfall der Chasaren deutlich: Die Alanen könnten, „wenn sie wollen, plündern, großen Schaden anrichten und bei den Chazaren große Not verursachen“, so hieß es dort. Doch die Alanen waren nicht die einzigen Gegner, genannt werden weitere potentielle Feinde, u. a. Nachkom-

22 Vgl. u.a. „Ioann Gotskij“, in: Kogonašvili (1995), 93f. Daher resultiert wohl auch die Auffassung, Johannes hätte am Konzil von Hiereia teilgenommen, das 754 von Kaiser Konstantin V. einberufen und auf dem die Bilderverehrung verurteilt worden war. Eine abweichende Position vertritt Vasiliev (1936), $89 \mathrm{f}$.

23 Pletnjowa (1978), 125.

24 Albrecht u. Herdick (2013), 31.

25 Vgl. die französische Übersetzung bei Auzépy (2006). Für die russische Version siehe beispielsweise Vasil'evskij (1878).

26 Vgl. Vinogradov (2010), der auf viele Unklarheiten in dieser Quelle hinweist, sowohl in Hinblick auf die Rekonstruktion der Ereignisse als auch auf die Übersetzungen aus dem Griechischen. 27 Müller L. (2001), 79/965. 
men der turkstämmigen Protobulgaren. ${ }^{28}$ Gemessen an der Verweildauer und dem Grad der Durchdringung der Halbinsel mit administrativen Strukturen muss die Herrschaft des Chaganats dennoch als besonders erfolgreich eingeschätzt werden, hatte sie doch für ca. drei Jahrhunderte eine gewisse Stabilität in der Region generiert.

28 Belke u. Soustal (1995), $87 \mathrm{f}$. 
